\title{
Service and Resource Differentiation in Shared-Path Protection Environments to Maximize Network Operator's Revenues
}

\author{
L. Velasco, M. Ruiz, J. Perelló, S. Spadaro, and J. Comellas
}

\begin{abstract}
Network operators are facing hard competition for opportunities in the telecommunications market, forcing network investments to be carefully evaluated before the decision-making process. A great part of core network operators' revenues comes from the provisioned connectivity services. Taking this premise as our starting point, we first examine the provisioning of differentiated services in current shared-path protection (SPP) environments. This analysis reveals that current resource assignment policies are only able to provide a very poor grade of service to the supported best-effort traffic. Aiming to improve this performance, a novel resource partitioning scheme called diff-WS is proposed, which differentiates those wavelengths supporting each class of service in the network. As a major goal of this paper, the benefits of diff-WS over current resource assignment policies are assessed from an economic perspective. For this purpose, the network operator revenues maximization (NORMA) problem is presented to design the optical network such that the operator's revenues are maximized. To solve NORMA, we derive statistical models to obtain, given a certain grade of service, the highest traffic intensity for each class of service and resource partitioning scheme. These models turn NORMA into a nonlinear problem, which is finally addressed as an iterative approach, solving an integer linear programming (ILP) subproblem at each iteration. The obtained numerical results on several network topologies illustrate that diff-WS maximizes resource utilization in the network and, thus, the network operator's profit.
\end{abstract}

Index Terms-Optical networks; Resource differentiation; Shared path protection.

\section{INTRODUCTION}

$\mathbf{T}$ he severe competition among network operators for attracting new clients forces them to cut the prices of the offered services, even at the risk of reducing the turnovers if the amount of service does not rise. In particular, the provisioned connectivity services represent a significant part of a core network operator's revenues, and a good way to increase them is by meeting a wider range of client necessities through service differentiation. For instance, many companies do not consider Internet access as a critical service. Therefore, the price that they are willing to pay for it is generally low.

Manuscript received July 19, 2010; revised October 15, 2010; accepted November 5, 2010; published January 26, 2011 (Doc. ID 131858).

The authors are with the Advanced Broadband Communications Center (CCABA), Universitat Politècnica de Catalunya (UPC), Barcelona, Spain (e-mail: lvelasco@ac.upc.edu).

Digital Object Identifier 10.1364/JOCN.3.000117
In contrast, business traffic is usually associated with strict service level agreements (SLAs), making this service much more expensive.

Typically, SLA contracts contain, among other parameters, the connection availability, that is, the probability that a connection will be operative at a random point in time. This connection availability is improved in dense wavelength division multiplexing (DWDM) networks by implementing protection and restoration schemes. Specifically, the shared-path protection (SPP) scheme has received much research attention, as it provides the best balance between availability, recovery time, and resource utilization (e.g., see [1,2]).

Basically, SPP consists of providing two disjoint paths between source and destination nodes, where the working path is replaced by the backup path upon a failure affecting the former. The same backup resource (i.e., wavelength channel) may be shared so as to provide protection to multiple working paths, as long as they are mutually diverse. Note that backup paths are configured to recover working paths from failures, but are not used under normal conditions. Hence, this unused backup capacity can be used for supporting extra traffic, which is preempted in case of a working path failure [3,4].

In this paper we propose a novel scheme to maximize those revenues resulting from the provisioning of differentiated services in DWDM networks. Two classes of service are defined: the SPP-based protected class (SP) and the besteffort preemptable class (BE). ${ }^{1}$ In the proposed scheme, we differentiate those resources dedicated for serving working SP traffic from those reserved for protection. Hence, we call this scheme as differentiated wavelength set (hereafter diff-WS), in contrast to the traditional unpartitioned wavelength set where all resources are shared by working and backup paths (sh-WS) [1,2]. In our previous work [5], the feasibility of the proposed diff-WS partitioning scheme was experimentally assessed in the CARISMA network test bed [6]. In this paper, we introduce analytical and statistical formulas to model the diff-WS and sh-WS schemes in mesh networks and compare them taking the expected revenues as the figure of merit.

Several proposals providing traffic differentiation, without resource differentiation, can be found in the literature. For example, the authors of $[7,8]$ suggest four service levels based on different recovery mechanisms in a mesh network. Although only two classes of service are considered in this work, the

\footnotetext{
${ }^{1}$ We assume that the best-effort client traffic (e.g., IP traffic) is transported by means of preemptable extra traffic resources on the DWDM network. Then, the terms best effort and extra traffic are equivalent in this paper.
} 
ideas here introduced can be easily extended to a higher number of classes. In this regard, some guidelines are pointed out throughout the following sections.

The authors of [9] define a new protection scheme and three resource types: (1) primary resources that can be used by primary paths, (2) spare resources that can be shared by backup paths, and (3) mixed resources that can be shared by both primary and backup paths. Although in this work we also separate primary and backup resources, our objective is to maximize the amount of served traffic and, as a result, the network operator revenues. Some other works have used different criteria to compare alternative architectures in DWDM networks. For instance, the authors of [10] addressed the maximization of revenues from the perspective of the SLA penalties, as SLA breaches represent large revenue losses for network operators. Our approach, however, consists of partitioning the set of available wavelengths per link, leading to differentiated sets of resources for each class of service and decoupling traffic dependences.

The remainder of this paper is organized as follows. Section II reviews the support of extra traffic in current SPP scenarios. Next, the diff-WS partitioning scheme is introduced, showing its advantages and drawbacks. Section III states the NORMA problem, whose objective is to maximize the revenues that result from serving differentiated traffic, while meeting certain grade of service (GoS) levels specified in terms of network blocking probability. Since the network traffic intensity plays a key role in NORMA, Section IV provides accurate statistical models to compute it for SP and BE traffic classes when the diff-WS or the $s h-W S$ scheme is used. In Section V, the NORMA integer linear programming (ILP) model, together with an iterative procedure to design the network under a given resource partitioning scheme, is presented. The derived ILP and statistical models are applied to different reference network topologies in Section VI, illustrating that the expected revenues are drastically increased if diff-WS is implemented. Finally, Section VII concludes the paper.

\section{WaVelength Partitioning Schemes}

\section{A. Best-Effort Traffic and SPP}

The topology of an optical network can be represented by a graph $G(N, E, W L)$, where $N$ represents the set of nodes, $E$ the set of links, and $W L$ the set of available wavelengths in each link, with size $W$. Assuming that wavelength conversion is not possible in the network, forcing the connections to satisfy the wavelength continuity constraint, we can split the graph $G$ into $W$ independent subgraphs $G^{i}(N, E, i)$, one per wavelength. Each subgraph $G^{i}$ represents the network connectivity through wavelength $i$, that is, $G(N, E, W L)=\left\{G^{i}\right.$ | $i \in W L\}$.

As mentioned before, SPP achieves high resource efficiency by sharing the backup resources where possible. This resource sharing is attained by reserving the backup resources but postponing their allocation until the working path fails. SP connection requests are routed on subgraphs $G^{i}$. Therefore, in order to assign a wavelength to both working and backup (a) SP connections

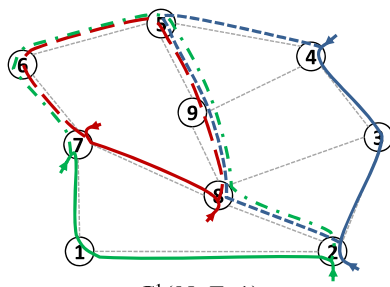

$G^{1}(N, E, 1)$

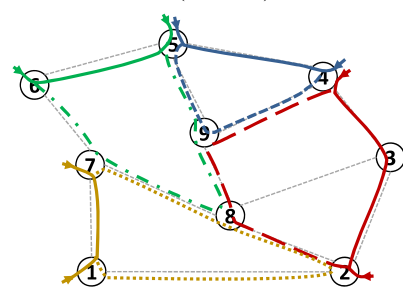

$G^{2}(N, E, 2)$
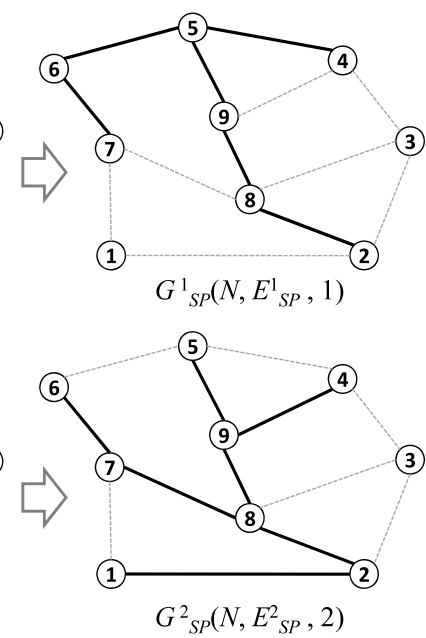

(b) Connectivity for BE traffic

Fig. 1. (Color online) Example of resource availability for BE traffic in two different wavelengths. Some SP connections have been established in (a). Solid color lines represent working paths, whereas dotted ones represent backup paths. The equivalent connectivity for BE traffic is shown with solid lines in (b).

paths, enough resources must exist for them. As a result, working and backup paths might interfere between them, since they might compete for the same resources. To illustrate this, Fig. 1(a) shows a number of SP connections established on two subgraphs, $G^{1}$ and $G^{2}$, where the same wavelength has been assigned to the working and backup paths for simplicity. In this example, a new connection 3-9 cannot be established.

Conversely, BE connections are established using only resources reserved for the backup paths that are currently idle. Therefore, BE traffic is subordinated to the SP traffic. In other words, while the SP traffic blocking probability $\left(P b_{S P}\right)$ depends on the network topology $G$ and the offered load, that of the BE traffic $\left(P b_{B E}\right)$ also depends on the amount of resources loaned by the currently established SP connections. Furthermore, as BE connections are borne on reserved backup resources, they are torn down abruptly when the resources supporting them are unallocated, even if their requested holding time $(h t)$ has not expired yet. In order to show this, we will assume in Fig. 1(a) that SP connection 1-7 is torn down. This would lead to the release of link 1-2 in $G^{2}$, forcing the teardown of any BE connection supported on such a resource. In this context, we define the billable time $(b t)$ of a connection as the total time being operative. Thus, $b t=\rho \cdot h t$, where $\rho$ is the proportion of consumed $h t$ over the total. Note that $\rho_{B E} \leq$ 1 as a consequence of the anticipated connection releases, whereas $\rho_{S P}=1$.

Aiming to quantify $P b_{B E}$, let us consider that a certain number of SP connections are established in the network. In this scenario, we denote $G_{S P}^{i}\left(N, E_{S P}^{i}, i\right)$ as the subgraph representing the resources available for BE traffic at wavelength $i$. Hence, $G_{S P}\left(N, E_{S P}, W L\right)=\left\{G_{S P}^{i} \mid i \in W L\right\}$, as shown in Fig. 1(b).

In fact, $P b_{B E}$ can be described as the contribution of two different factors: the connectivity of the graph where the routes are computed (note that in the case of $\mathrm{BE}$ connections, 
subgraphs $G_{S P}^{i}$ could not be connected) and the offered load to the network. For the ongoing analysis, let $R_{B E}$ be the ordered set of $\mathrm{BE}$ connection requests and $R_{B E}^{i}\left(G_{S P}^{i}\right)$ be the subset of $R_{B E}$ having a feasible route over $G_{S P}^{i}$. Then, the complete set of $\mathrm{BE}$ connection requests with a feasible route on $G_{S P}$ is given by

$$
R_{B E}^{*}=\bigcup_{\forall i \in W L} R_{B E}^{i}\left(G_{S P}^{i}\right)
$$

Moreover, we define $P\left(R_{B E}, G_{S P}\right)$ as the probability that a $\mathrm{BE}$ connection request can be established subject to the current $G_{S P}$ connectivity, which can be expressed as

$$
P\left(R_{B E}, G_{S P}\right)=\frac{\left|R_{B E}^{*}\right|}{\left|R_{B E}\right|} .
$$

Therefore, we can formulate $P b_{B E}$ as the sum of two terms. The first one, capturing the graph's unconnectivity, is the number of $\mathrm{BE}$ connection requests without a feasible route on $G_{S P}$ over the size of $R_{B E}$. The second one, capturing the lack of resources, is the number of $\mathrm{BE}$ connection requests with a feasible route on $G_{S P}$ weighted by a probability function that depends on $G$ and the offered loads of SP $\left(I_{S P}\right)$ and BE $\left(I_{B E}\right)$ traffic, also over the size of $R_{B E}$. This is expressed in Eq. (3). As shown, $P\left(R_{B E}, G_{S P}\right)$ leads to a lower bound for $P b_{B E}$ :

$$
\begin{aligned}
P b_{B E} & =\frac{\left|R_{B E}\right|-\left|R_{B E}^{*}\right|}{\left|R_{B E}\right|}+f\left(G, I_{S P}, I_{B E}\right) \cdot \frac{\left|R_{B E}^{*}\right|}{\left|R_{B E}\right|} \\
& \geq 1-P\left(R_{B E}, G_{S P}\right) .
\end{aligned}
$$

Aiming to illustrate such a lower bound for $P b_{B E}$, let us assume that $R_{B E}$ contains all possible node pairs (i.e., uniform traffic distribution). For each node pair, we investigate whether a feasible route exists on $G_{S P}$. For example, node pairs 7-5 and $4-1$ in Fig. 1 can be connected through feasible routes (e.g., 7-6-5 in $G_{S P}{ }^{1}$ and $4-9-8-2-1$ in $G_{S P}{ }^{2}$ ), whereas pair 7-3 cannot. Applying now Eq. (2), $P\left(R_{B E}, G_{S P}\right)$ equals 0.78, with $P b_{B E}$ having a lower bound equal to 0.22 , that is, as a result of the $G_{S P}$ unconnectivity. In conclusion, the BE traffic GoS will generally be very poor due to its dependence on the $\mathrm{SP}$ traffic, thus leading to very low BE traffic revenues.

\section{B. Proposed Wavelength Partitioning Scheme}

In view of the above, we propose a novel wavelength partitioning scheme for provisioning differentiated traffic in DWDM transport networks. Our main objective is to improve the $\mathrm{BE}$ traffic performance and, as a result, the expected network revenues.

To this end, we split the complete set of wavelengths into two different subsets of size $W / 2$, namely, $W L_{S P}$ and $W L_{B E}$, dedicated to $\mathrm{SP}$ working and backup path reservations, respectively. The wavelengths in both sets are rigidly related as follows. Being an SP working path assigned to wavelength $i$, its backup path is assigned to wavelength $W-i$. Figure 2 shows an example with four wavelengths per link, carrying the same traffic as in Fig. 1. We call this wavelength partitioning scheme differentiated wavelength set (diff-WS), in contrast to the
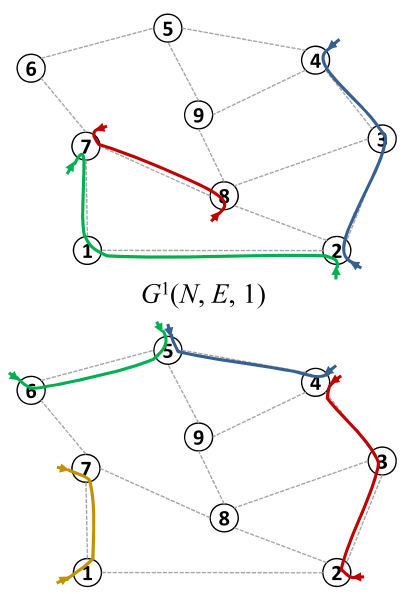

$G^{2}(N, E, 2)$ (a) Working SP paths (b) Protecting SP path reservations
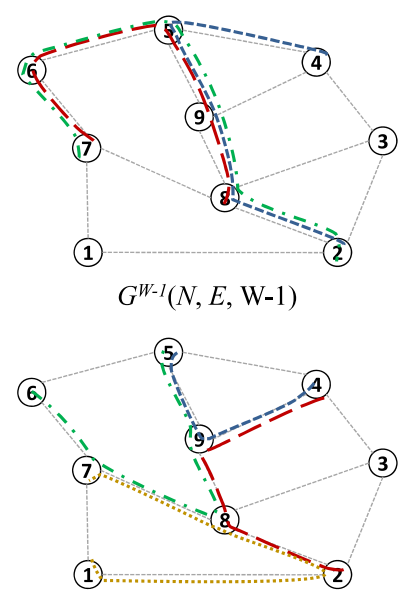

$G^{\mathrm{W}-2}(N, E, \mathrm{~W}-2)$
Fig. 2. (Color online) Example of diff-WS where some SP connections have been established. (a) SP working paths (solid color lines); (b) SP protecting paths (dotted color lines).

TABLE I

RWA ALGORITHM FOR SP CONNECTIONS

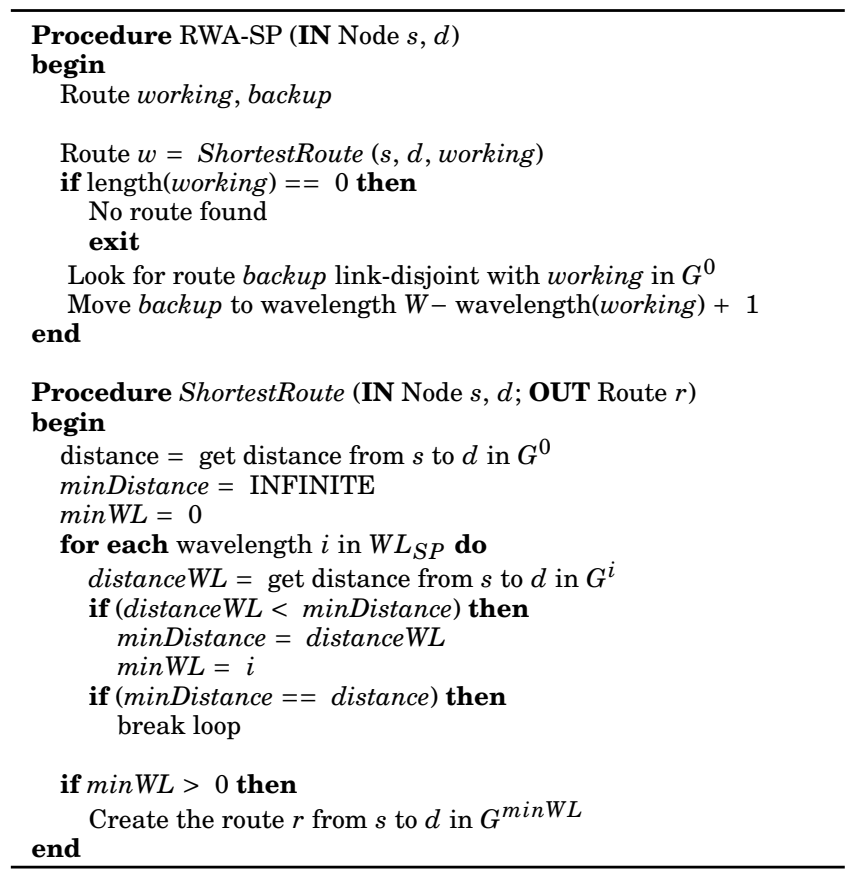

traditional unpartitioned wavelength set where all resources are shared by working and backup paths (sh-WS) [1,2], as detailed in the previous subsection.

In particular, backup resource sharing in diff-WS is restricted to those connections whose working paths are assigned the same wavelength. For this purpose, an algorithm that computes two link-disjoint paths jointly, considering those wavelengths in $W L_{B E}$ as unused, would be required. As an example, Table I describes an algorithm adapted from [5], where $G^{0}$ is the graph describing the network physical topology. The algorithm finds the shortest route for the 


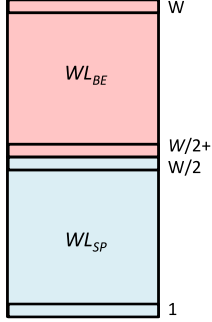

(a)

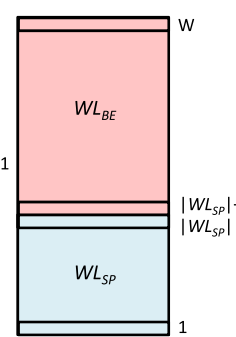

(b)

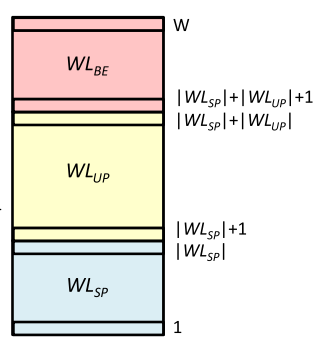

(c)
Fig. 3. (Color online) Conceptual representation of a DWDM link: (a) symmetrical diff-WS, (b) asymmetrical diff-WS, and (c) three-class diff-WS

working path, assigns a wavelength within $W L_{S P}$, and finds a link-disjoint path.

It shall also be highlighted that the whole $W L_{B E}$ set in diff-WS is dedicated to backup path reservations. Hence, it can be entirely used to support BE traffic. This makes diff-WS more beneficial than $s h$-WS, where only those resources already reserved for backup SP paths can be used for carrying $\mathrm{BE}$ traffic. As will be shown in Section VI, this new approach provides optimum GoS for BE traffic. In fact, this is achieved in certain network topologies at the expense of slightly lowering the amount of SP traffic served. Nonetheless, the total network revenues from both kinds of services are drastically leveraged when diff-WS is applied.

Furthermore, the diff-WS partitioning scheme can be used under a wide set of traffic scenarios by appropriately tuning the size of the $W L_{S P}$ and $W L_{B E}$ sets. Figure 3 shows a conceptual representation of a DWDM link. A symmetrical diff-WS partitioning such as that represented in Fig. 3(a) becomes suitable when $I_{S P} \geq I_{B E}$, whereas the asymmetrical diff-WS partitioning shown in Fig. 3(b) fits better when $I_{S P}<I_{B E}$. Note that $\left|W L_{B E}\right| \geq\left|W L_{S P}\right|$ is necessary to provide protection to SP connections. Moreover, the size of the wavelength sets can be dynamically managed to adapt the network to traffic fluctuations. Finally, a third class of service, e.g., the unprotected class (UP), has been defined in Fig. 3(c), and thus a new set $W L_{U P}$ has been created. In particular, this paper focuses on the symmetrical configuration with the two classes of traffic depicted in Fig. 3(a).

\section{NORMA PROBLEM STATEMENT}

As stated in the introduction, connectivity services become the core business for network operators. Therefore, any investment related to their deployment must be carefully analyzed, as it may critically impact on their final profit. In this section, we propose the network operator revenues maximization (NORMA) problem, which allows the optimal network design that maximizes the expected revenues under a certain resource partitioning scheme, such as sh-WS or diff-WS, to be found. It is worth noting that real backbone optical transport network topologies (e.g., [11]) share planarity as a common characteristic. Moreover, to provide protection, 2-connectivity is another requirement for feasible topologies.
The NORMA problem can be formulated as follows:

Given:

(a) the physical network topology represented by a graph $G(N, E, W L)$, with $N$ being the set of locations where a node must be placed, $E$ being the set of already-deployed optical fibers, and $W L$ being the set of wavelengths in each optical link;

(b) a set $S$ of classes of service to be provided, index $j$;

(c) the network operator's pricing structure, specified by a fixed fee $C_{j}$ charged to the customers per time unit of class of service $j$;

(d) the blocking probability threshold $P b_{j}^{\max }$ allowed for each class of service $j$;

(e) a set $K$ of wavelength partitioning schemes, index $k$. In this paper we assume $K=\{s h-W S$, diff-WS $\}$.

\section{Output:}

(a) a wavelength partitioning scheme $k$;

(b) the set of links $E(k)$ in the network designed for the partitioning scheme $k$ ensuring that the resulting topology is planar and, at least, 2-connected;

(c) the maximum traffic intensity for the given classes of service unleashing $P b_{j}^{\max }$ thresholds.

Objective: Maximize the expected revenues for the designed network coming from serving the maximum amount of traffic belonging to the defined classes. Therefore, the NORMA objective function can be expressed as

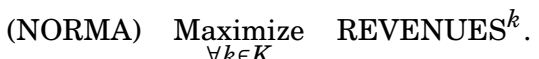

The revenues model consists of defining optical connections belonging to class of service $j$, providing for each one the service level specified in the SLA. One important parameter in the SLA is the availability of the connection. As previously discussed, protection or restoration schemes can be implemented to increment the availability of the connections. In this study, we assume that only two classes of service have been put on the market: the shared protected (SP) class, which uses shared protection to provide a highly available service, and the best-effort (BE) class, which uses protecting resources to provide extra traffic as long as those resources are not requested (i.e., failure-free conditions). Thus, $S=\{\mathrm{SP}, \mathrm{BE}\}$.

The revenues from selling these services can be computed knowing the billable time. We define $b t_{j}^{k}$ as the billable time of service class $j$ during a certain time interval $\Delta t$ (e.g., 1 year) using wavelength partitioning scheme $k$. Note that such a time can be computed as the amount of expected arrivals during $\Delta t$ multiplied by the average connection $h t$. Therefore, defining $i a t_{j}^{k}$ as the average interarrival time, $b t_{j}^{k}$ can be formulated as

$$
\begin{aligned}
b t_{j}^{k} & =|N| \cdot \frac{\Delta t}{i a t_{j}^{k}} \cdot\left(1-P b_{j}\right) \cdot\left(h t_{j}^{k} \cdot \rho_{j}^{k}\right) \\
& =|N| \cdot I_{j}^{k} \cdot \rho_{j}^{k} \cdot\left(1-P b_{j}\right) \cdot \Delta t,
\end{aligned}
$$


TABLE II

Characteristics of THE ANALYzed Networks

\begin{tabular}{llll}
\hline & Min. & Max. & Mean \\
\hline$|N|$ & 9 & 28 & 19 \\
$|E|$ & 11 & 44 & 28 \\
$\delta$ & 2.44 & 3.37 & 2.90 \\
$h$ & 1.86 & 4.07 & 2.93 \\
\hline
\end{tabular}

where $I_{j}^{k}=h t_{j}^{k} / i a t_{j}^{k}$ represents the offered load per node belonging to class of service $j$ when wavelength partitioning scheme $k$ is used. Then, the revenues obtained from serving the two classes of service over an optical network using wavelength partitioning scheme $k$ can be computed as

$$
\begin{aligned}
\text { REVENUES }^{k} & =\sum_{\forall j \in S} b t_{j}^{k} \cdot C_{j} \\
& =\sum_{\forall j \in S}\left(|N| \cdot I_{j}^{k} \cdot \rho_{j}^{k} \cdot\left(1-P b_{j}\right) \cdot \Delta t \cdot C_{j}\right) .
\end{aligned}
$$

In light of Eqs. (5) and (6), the maximization of revenues is equivalent to maximize the offered load while still meeting the blocking probability threshold. To the best of our knowledge, no intensity models for dynamic traffic over mesh networks, suitable to be used in mathematical programming models, can be found in the literature. Therefore, we face this problem from a statistical viewpoint. To this end, in the next section we focus on predicting the offered load to the network by unleashing $P b_{j}^{\max }$ based on simple network characteristics.

\section{TRAFFiC Intensity Statistical Models}

Inspecting Eq. (6), two unknowns can be identified: the offered load and the actual proportion of provided $h t$. In this section, we focus on modeling these variables from a statistical point of view, referring to $I_{j}^{k}$ and $\rho_{j}^{k}$ as the response variables to be modeled.

Aiming to obtain likely values for the response variables, we have studied their behavior over a meaningful set of networks. These network scenarios were chosen from the set of real backbone optical transport networks presented in [11]. From the complete set, a subset of 16 biconnected and planar networks was selected, covering in this way a wide range of distinct topologies. Each network was identified by means of 12 topology-dependent characteristics (independent variables) that were candidates to be part of the model. To illustrate the range of the sample, Table II summarizes four of these characteristics: $|N|,|E|$, the average nodal degree $(\delta)$, and the average path length in hops $(h)$.

We conducted a large amount of simulations over the networks under study for every traffic class and wavelength partitioning scheme. In such experiments, each DWDM link was equipped with 16 wavelengths, and no wavelength conversion capabilities were provided to the optical nodes. For $s h$-WS, the CAFES algorithm in [1] was adapted to compute feasible routes for the SP connections while satisfying the wavelength continuity constraint. In contrast, the algorithm in Table I was used for diff-WS. In this regard, $P b_{S P}^{\max }=1 \%$ was assumed for the SP class, a value largely used in the literature.
In contrast, a significantly higher $P b_{B E}^{\max }=5 \%$ was assumed for the BE class.

Every simulation for a specific traffic class and wavelength partitioning scheme resulted in a tuple containing the offered load to the network, the blocking probability, and the proportion of $h t$ served. We chose those tuples with $P b$ equal to $P b_{j}^{\max }$. Then, together with the candidate set of independent variables, we applied a multiple linear regression to obtain statistical models for the response variables.

Assuming nonlinear relations among the candidate independent variables, we used a logarithmic transformation for the response and independent variables, enabling a linear regression methodology. Moreover, aiming to avoid overfitting, which could be a drawback in further predictions, we limit the number of independent variables in the models to be two.

Regarding the traffic intensity, four models are needed, one per $I_{j}^{k}$. From the results, we observed the best fit using $|E|$ and $h$ as independent variables. The former provides the amount of network resources (i.e., the network capacity), whereas the latter gives information about the amount of resources per connection. We also concluded that a general parametrical formula with those independent variables can be used with specific linear coefficient values for each $I_{j}^{k}$ model. Equation (7) shows the prediction model where the logarithmic transformation has been reversed, so that the linear coefficients become exponents. Besides, Table III shows the values of the exponents for each $I_{j}^{k}$ model.

$$
I_{j}^{k}=\frac{10^{\alpha(k, j)} \cdot|E|^{\beta(k, j)}}{h^{\gamma(k, j)}} \pm \varepsilon(k, j) .
$$

In order to provide a confidence interval to the statistical models, the parameter $\varepsilon(k, j)$ collecting the relative error has also been included. As shown in Table III, these errors are close to $5 \%$, except for the BE traffic class with sh-WS, where fewer networks were available for the model (a discussion about this case is given below). Moreover, the values of the Pearson coefficient $\left(R^{2}\right)$ are higher than $95 \%$ as shown in Table III, thus giving a tight fit for the offered load models.

At this point, we take up again the blocking probability bound (1-P( $\left.R_{B E}, G_{S P}\right)$ ) defined in Eq. (3); if that bound is higher than $P b_{j}^{\max }$, the traffic intensity must be set to zero since any traffic load would not satisfy the requested GoS.

However, there is only one case where this may happen, namely, the case of the $\mathrm{BE}$ traffic under $s h-W S$ as a consequence of its subordination to the SP traffic. In view of this, the $I_{B E}^{s h-W S}$ model needs to be completed with an expression to predict whether $\left(1-P\left(R_{B E}, G_{S P}\right)\right)>P b_{B E}^{\max }$. We have computed $P\left(R_{B E}, G_{S P}\right)$ for every network under study, finding a tight relation between $P\left(R_{B E}, G_{S P}\right)$ and $h$. We observed $\left(1-P\left(R_{B E}, G_{S P}\right)\right)>P b_{B E}^{\max }$ in every network with $h>2.5$. Therefore, the $I_{B E}^{s h-W S}$ model becomes a discontinuous function, where Eq. (7) models $I_{B E}^{s h-W S}$ provided that $h \leq 2.5$; otherwise $I_{B E}^{s h-W S}=0$.

Regarding the proportion of provided $h t$, only the model for the particular case of BE traffic under $s h$-WS is finally needed. By simple inspection of the simulation results, we concluded 
TABLE III

PARAMETERs AND OBSERVEd Adjustments For the InTensity Models

\begin{tabular}{lllllll}
\hline Scheme $(k)$ & Class $(j)$ & $\alpha$ & $\beta$ & $\gamma$ & $\varepsilon(\%)$ & $R^{2}(\%)$ \\
\hline sh-WS & SP & 0.222 & 0.931 & 3.07 & 5.62 & 99.2 \\
& BE & 1.522 & 0 & 6.40 & 9.94 & 95.1 \\
diff-WS & SP & 0.334 & 0.724 & 2.72 & 54 & 9.48 \\
& BE & 0.548 & 0.603 & 2.56 & 9.48 \\
\hline
\end{tabular}

that $\rho_{B E}^{s h-W S} \approx 0.6$. For the rest of the models $\rho_{j}^{k}=1$, thus reflecting the independence between traffic classes.

Once the statistical models for the response variables have been obtained and their goodness of fit assessed, we use them to solve the NORMA problem previously formulated.

\section{NORMA ItERATIVE Method AND ILP MODEL}

In view of the intensity model in Eq. (7) and the revenues expression in Eq. (6), we can reformulate the NORMA objective function (i.e., removing constants, grouping terms, etc.) as

$$
\text { Maximize REVENUES }{ }^{k} \equiv \text { Maximize } \sum_{\forall j \in S} \frac{\theta_{j}^{k} \cdot|E|^{\beta(k, j)}}{h^{\gamma(k, j)}}
$$

for a given partitioning scheme $k$, where $\theta_{j}^{k}$ is a positive constant. Looking at Eq. (8), we are facing a nonlinear problem. Nevertheless, Proposition 1 allows us to define an iterative method where a linear problem is solved at each iteration.

Proposition 1. Let $G_{1}\left(N, E_{1}\right)$ and $G_{2}\left(N, E_{2}\right)$ be two graphs with $\left|E_{1}\right|$ and $\left|E_{2}\right|$ links and with minimum $h_{1}$ and $h_{2}$, respectively $\left(G_{1}\right.$ and $G_{2}$ are optimal with respect to $\left.h\right)$. If $\left|E_{2}\right|=\left|E_{1}\right|+1$, then for a given scheme $k$,

$$
\sum_{\forall j \in S} \frac{\theta_{j}^{k} \cdot\left|E_{2}\right|^{\beta(k, j)}}{h_{2}^{\gamma(k, j)}}>\sum_{\forall j \in S} \frac{\theta_{j}^{k} \cdot\left|E_{1}\right|^{\beta(k, j)}}{h_{1}^{\gamma(k, j)}} \quad \begin{aligned}
& \forall \beta(k, j)>1 \\
& \forall \gamma(k, j)>1,
\end{aligned}
$$

where $\beta(k, j)$ and $\gamma(k, j)$ become parameters greater than 1 for each scheme and class of service.

Proof. If $h_{1}$ is the minimum for every network with $\left|E_{1}\right|$ links, optimizing for $\left|E_{2}\right|=\left|E_{1}\right|+1$ links will result in a graph $G_{2}\left(N, E_{2}\right)$ with average path length $h_{2} \leq h_{1}$, provided that a feasible solution exists.

As a consequence of Proposition 1, the NORMA problem can be solved iteratively by fixing the number of links $a$ at each iteration. In this way, a linear problem is solved, since $|E|$ remains constant and the objective function to minimize is $h$.

It is worth mentioning that each linear problem finds the 2-connected and planar topology with $a$ number of links that minimizes $h$. Therefore, the optimal solution of the NORMA problem is obtained with the maximum number of links and the minimum $h$.

To solve NORMA the following sets and parameters are defined:
TABLE IV

NORMA ITERATIVE METHOD

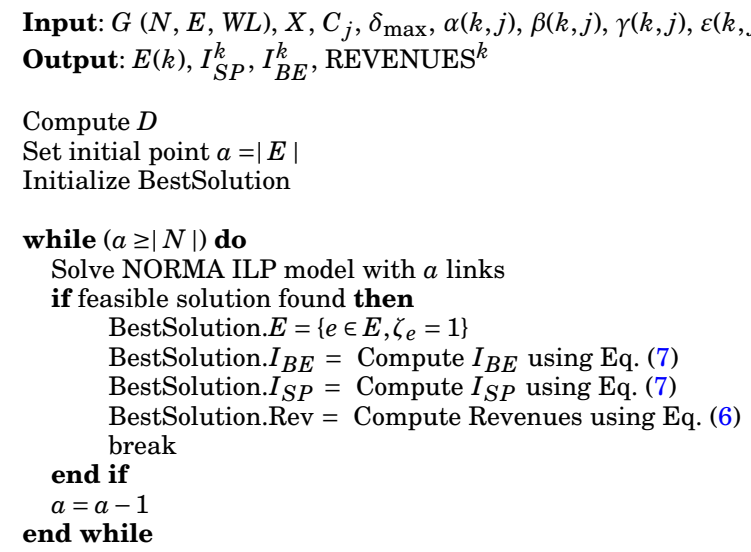

return BestSolution

$N \quad$ set of nodes, index $n$

$E \quad$ set of links, index $e$

$\Omega(n) \quad$ set of incident links on node $n$

$K \quad$ set of partitioning schemes, index $k$

$D \quad$ set of suitable source-destination pairs $\left\{s_{d}, t_{d}\right\}$, index $d$.

$X \quad$ set of exclusions to ensure planarity, index $x$

$\phi_{e}^{x} \quad 1$ if link $e$ is in the exclusion $x, 0$ otherwise

$\delta_{\max } \quad$ maximum nodal degree

$a \quad$ desired number of links

$M \quad$ a large positive constant.

Additionally, the following variables are defined:

$\zeta_{e} \quad$ binary, 1 if link $e$ is in the designed network, 0 otherwise

$\omega_{e}^{d} \quad$ binary, 1 if the source-destination pair $d$ uses link $e$ for the shortest path, 0 otherwise

$\kappa_{e}^{d} \quad$ binary, 1 if the source-destination pair $d$ uses link $e$ for the alternative path, 0 otherwise.

We use the set $D$ to ensure that two link-disjoint routes can be found in the designed network topology (2-connectivity condition). Note that if $D$ does not contain all node pairs, one (or more) 2-connected subgraph(s), containing the source-destination nodes, will be obtained instead.

The iterative method starts fixing the parameter $a$ to the maximum number of links, reducing this number until a feasible solution is found. Table IV shows the NORMA iterative method to obtain the optimal solution for a given partitioning 

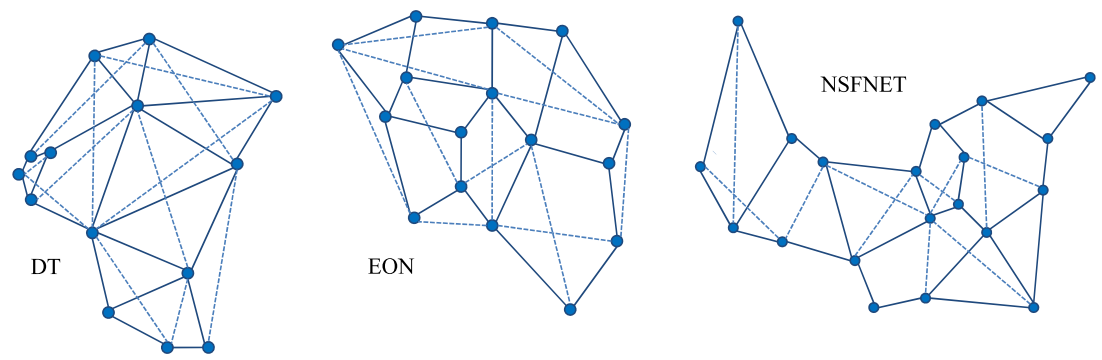

\begin{tabular}{|c|c|c|c|c|}
\cline { 2 - 5 } \multicolumn{1}{c|}{} & $|\boldsymbol{N}|$ & $|\boldsymbol{E}|$ & $\boldsymbol{\delta}$ & $\boldsymbol{h}$ \\
\hline DT & 14 & 23 & 3.29 & 2.34 \\
\hline EON & 16 & 23 & 2.87 & 2.64 \\
\hline NSFNET & 20 & 28 & 2.80 & 3.22 \\
\hline
\end{tabular}

\begin{tabular}{|c|c|c|c|c|}
\cline { 2 - 5 } \multicolumn{1}{c|}{} & $|\boldsymbol{N}|$ & $|\boldsymbol{E}|$ & $|\boldsymbol{X}|$ & $|\boldsymbol{D}|$ \\
\hline DT series & 14 & 35 & 5 & 91 \\
\hline EON series & 16 & 35 & 6 & 120 \\
\hline NSFNET series & 20 & 38 & 5 & 190 \\
\hline
\end{tabular}

Fig. 4. (Color online) Network topologies used for evaluation with their most relevant characteristics. Solid lines represent the topology's original links, whereas dotted lines are used for the added connectivity.

scheme. Finally, the NORMA ILP model for a fixed number of links $a$ can be formulated as follows:

$$
\text { (NORMA) minimize } h=\frac{1}{|D|} \sum_{\forall d \in D} \sum_{\forall e \in E} \omega_{e}^{d}
$$

subject to:

$$
\begin{aligned}
& \sum_{\forall e \in \Omega(n)} \omega_{e}^{d}=1 \quad \forall d \in D \quad \forall n \in\left\{s_{d}, t_{d}\right\} \\
& \sum_{\forall e \in \Omega(n)} \omega_{e}^{d} \leq 2 \quad \forall d \in D \quad \forall n \in N-\left\{s_{d}, t_{d}\right\}, \\
& \sum_{\substack{\forall e^{\prime} \in \Omega(n) \\
e^{\prime} \neq e}} \omega_{e^{\prime}}^{d} \geq \omega_{e}^{d} \quad \forall d \in D \quad \forall n \in N-\left\{s_{d}, t_{d}\right\} \forall e \in \Omega(n), \\
& \sum_{\forall e \in \Omega(n)} \kappa_{e}^{d}=1 \quad \forall d \in D \quad \forall n \in\left\{s_{d}, t_{d}\right\} \\
& \sum_{\forall e \in \Omega(n)} \kappa_{e}^{d} \leq 2 \quad \forall d \in D \quad \forall n \in N-\left\{s_{d}, t_{d}\right\}, \\
& \sum_{\substack{\forall e^{\prime} \in \Omega(n) \\
e^{\prime} \neq e}} \kappa_{e^{\prime}}^{d} \geq \kappa_{e}^{d} \quad \forall d \in D \quad \forall n \in N-\left\{s_{d}, t_{d}\right\} \forall e \in \Omega(n),(16) \\
& \omega_{e}^{d}+\kappa_{e}^{d} \leq 1 \quad \forall d \in D \quad \forall e \in E, \\
& \sum_{\forall d \in D}\left(\omega_{e}^{d}+\kappa_{e}^{d}\right) \leq M \cdot \zeta_{e} \quad \forall e \in E, \\
& \sum_{\forall e \in E} \zeta_{e}=a \\
& \sum_{\forall e \in E} \zeta_{e} \cdot \varphi_{e}^{x} \leq 1 \quad \forall x \in X \\
& \sum_{\forall e \in \Omega(n)} \zeta_{e} \leq \delta_{\max } \quad \forall n \in N .
\end{aligned}
$$

In the model, the objective function (10) indirectly maximizes revenues for the given number of links. Constraints (11)-(17) ensure that the designed topology is at least 2-connected, that is, a pair of link-disjoint routes must exist in the network for every source-destination pair. Constraint (18) stores the use of every link. Constraint (19) guarantees that the desired number of links $a$ is provided. Constraint (20) ensures that the topology is planar. Finally, constraint (21) limits nodal degrees.

The NORMA method and model were implemented in iLog-OPL and solved by the CPLEX v.11.0 optimizer [12] on a $2.4 \mathrm{GHz}$ Quad-Core machine with 8 GB RAM memory. The next section discusses the obtained results.

\section{Illustrative Numerical Results}

For evaluation, three backbone optical network series were created, starting from three well-known base topologies and adding extra connectivity. To this end, we used the moderately meshed 14-node Deutsche Telecom (DT) and 16-node European Optical Network (EON) topologies, in addition to a quite sparse 20-node NSFNET topology. These networks do not belong to the set of networks used to obtain the statistical models, so they can be used for evaluation. Figure 4 shows the resulting test topologies and reviews their most relevant characteristics.

Aiming to compare the traffic intensities under the $s h$-WS and diff-WS schemes, Fig. 5 plots their absolute values as a function of the number of links for every optimal network in each topology series. Focusing on the SP traffic class, the traffic intensity under diff-WS is higher than that under sh-WS for quite sparsely meshed networks, but lower for moderately meshed ones.

It is worth noting that the number of SP connections being established under the $s h$-WS scheme depends on the shareability degree of the protection resources, i.e., the number of protection paths sharing each resource. The higher this degree the more resources are kept available for working paths. The shareability degree depends on the existence of multiple disjoint working paths in the network, by definition of the SPP scheme, and thus on the network's mesh degree. Then, the available resources will be increased by increasing $\delta$. In contrast, the number of resources available for working paths under the diff-WS scheme is given by the fixed size of $W L_{S P}$, since differentiated resources are used for working and for protection paths. 

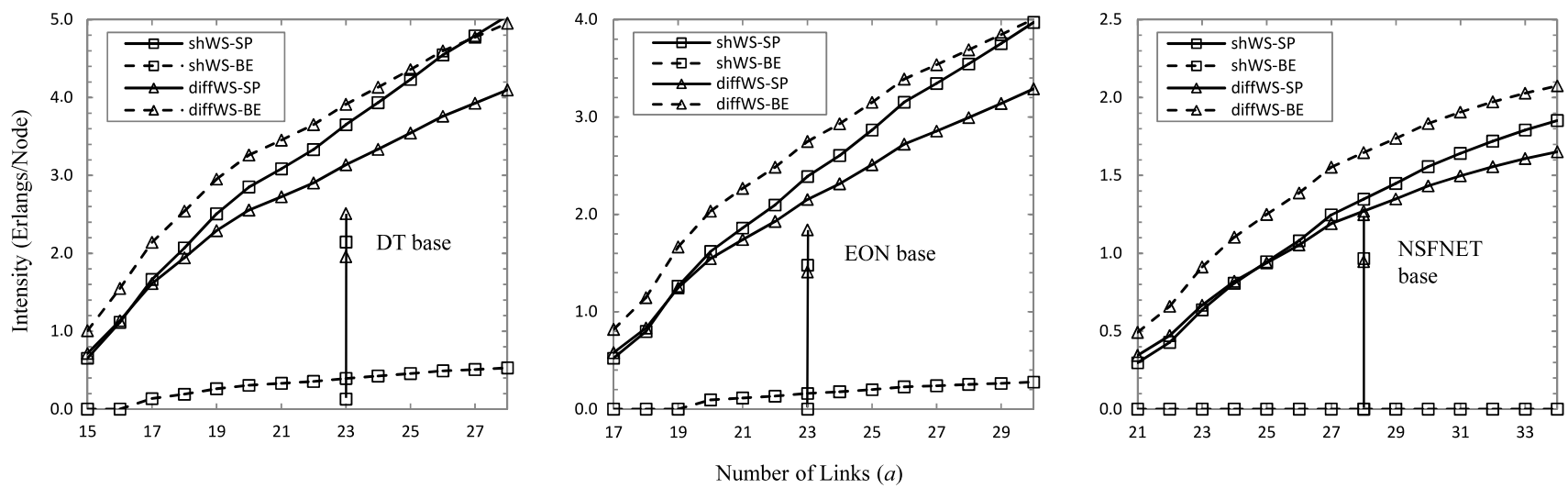

Fig. 5. Intensity against the number of links for the DT series (left), EON series (center), and NSFNET series (right).
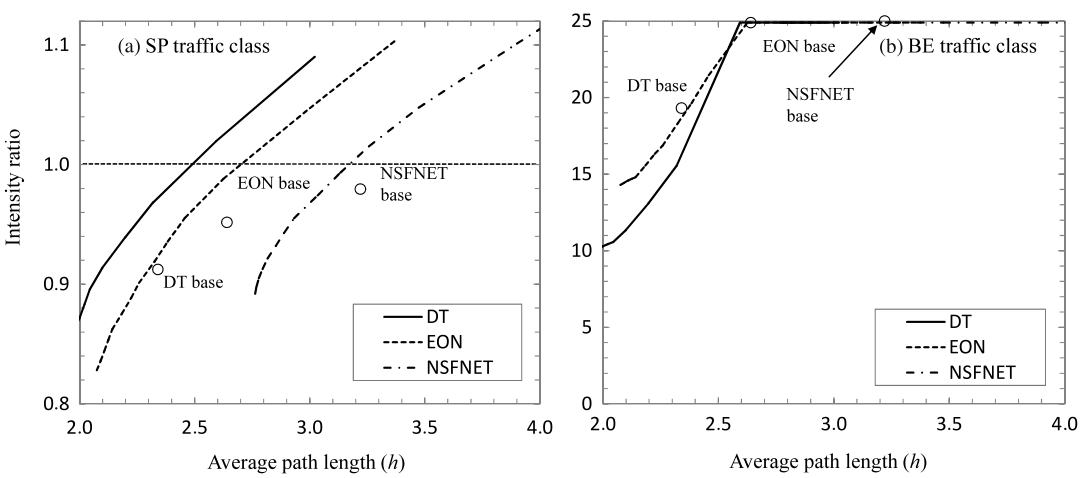

Fig. 6. Intensity ratio against the average path length for (a) SP and (b) BE traffic. Base topologies are also positioned. All networks support slightly more SP traffic under sh-WS but much more BE traffic under diff-WS.

For the BE traffic class, the obtained intensity is much higher under diff-WS as a consequence of resource differentiation. Note that the intensity becomes zero for those sparsely and moderately meshed networks with $h>2.5$. In fact, the $\mathrm{BE}$ traffic intensity is zero for each topology belonging to the NSFNET series.

Besides, the corresponding base topology is also positioned in each graph in Fig. 5. As seen, the traffic intensity values are clearly worse than those of the optimal network with the same number of links.

In order to compare the relative values of traffic intensity obtained under $s h$-WS and diff-WS, let us define their intensity ratio as $I_{j}^{\text {diff-WS }}: I_{j}^{s h-W S}$. An intensity ratio equal to 1 describes a network that can carry the same amount of traffic under both wavelength partitioning schemes; a value greater (lower) than 1 means more traffic being carried under diff-WS (sh-WS). Figure 6 shows this intensity ratio for SP and BE traffic as a function of $h$. Each plot represents the ratio values in the networks under study. Additionally, the base test topologies are also positioned.

Figure 6(a) shows that the sh-WS scheme supports more SP traffic intensity when applied to moderately and highly meshed networks (short average path lengths). Note that $\delta$ and $h$ are closely related. This is also the case for the DT, EON, and NSFNET base networks, only supporting 91\%, 95\%, and $98 \%$ of traffic under diff-WS with respect to $s h$-WS, respectively. In contrast, diff-WS supports more SP traffic when applied to sparsely meshed networks.

A similar study can be done for the BE traffic [Fig. 6(b)]. In this case, diff-WS allows more traffic than $s h$-WS regardless of the average nodal degree value. As observed, diff-WS can carry at least 10 times more traffic than diff-WS. For instance, the diff-WS scheme allows about 19.3 times more traffic than $s h$-WS to be carried over the DT base network. In fact, since most of the networks under study present $h>2.5$ hops, the $\mathrm{BE}$ traffic intensity becomes zero under $s h$-WS, resulting in infinite intensity ratios. This also happens in the EON and NSFNET base networks.

With aims to compare the revenues obtained under each partitioning scheme, Fig. 7 illustrates the evolution of the revenues increment percentage with diff-WS with respect to $s h$-WS as a function of the average nodal degree. As shown, although SP traffic intensity is lower under diff-WS, the finally obtained revenues are higher even in highly meshed network 


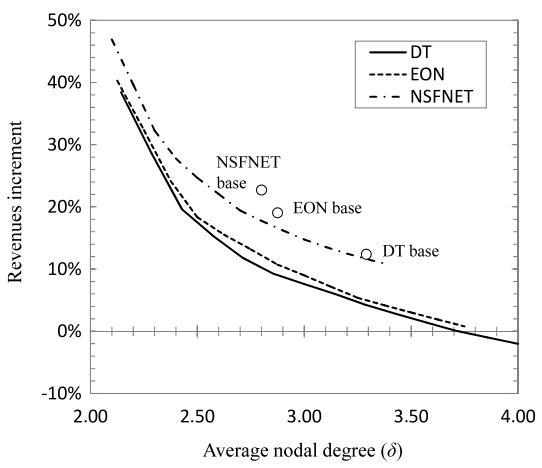

Fig. 7. Revenues increment (\%) against the average nodal degree for a price ratio $C_{S P}: C_{B E}=5: 1$.

topologies. As shown, only those optimal topologies in the DT series with $\delta>3.7$ lead to increased revenues with $s h$-WS.

In addition, the corresponding base topologies are also positioned in Fig. 7. Interestingly, the obtained percentages are much higher than those of the optimal network with the same number of links. Therefore, any nonoptimal network topology increases the revenues increment percentage with diff-WS against $s h$-WS; thus the former is more beneficial.

To compare the prices of $\mathrm{SP}$ and $\mathrm{BE}$ services, we also define the price ratio $C_{S P}: C_{B E}$. Note that a fair price ratio could be $5: 1$, reflecting the amount of resources used by each service, as suggested in [10]. Figure 8 plots the total revenues obtained with each wavelength partitioning scheme as a function of the price ratio for the base topologies. In the case of the DT network we observed the break-even price ratio at $12: 1$. In the case that a $5: 1$ price ratio would be applied, diff-WS would obtain $12.4 \%$ additional revenues than $s h$-WS. Applying a more aggressive price rate of $8: 1$ diff-WS would still obtain more revenues $(4.5 \%)$. As a result, the revenues from operating the DT network would still be higher in a wide range of price ratios if the diff-WS scheme is applied. A similar analysis can be done for the EON and NSFNET networks. In these cases, the break-even price ratio is higher than $20: 1$, being that the revenues from operating this network are higher with the
diff-WS scheme (19.1\% and $22.7 \%$ higher with $5: 1$ price ratio for EON and NSFNET, respectively).

\section{CONCLUSIONS}

This paper has studied current differentiated service provisioning in SPP environments. From an initial analysis, we found that the usual resource assignment policy leads to very poor GoS figures for the BE traffic class. Aiming to improve the $\mathrm{BE}$ service performance without deteriorating the higher-quality SP service, the diff-WS wavelength partitioning scheme was proposed. This scheme consists of assigning differentiated resources to each class of service. In particular, resource differentiation in DWDM networks is attained by defining one wavelength set for each class. In this way, the amount of BE traffic served can grow dramatically, without any meaningful SP traffic intensity reduction.

In order to compare the performance obtained by the evaluated partitioning schemes, the NORMA problem was presented. The objective of such a problem is the design of the optical network that maximizes network operator revenues from serving as much differentiated traffic as possible, while meeting certain GoS requirements. To this end, statistical models to compute the traffic intensity for the objective GoS were derived.

The considered wavelength partitioning schemes were compared solving the NORMA problem for three backbone optical network series. The sh-WS scheme was probed to support slightly more SP traffic intensity than diff-WS when applied to moderately and highly meshed networks, whereas diff-WS allows much more BE traffic than sh-WS. This fact leads diff-WS to provide the highest revenues even in highly meshed network topologies.

In light of the extensive evaluation, we are able to conclude that diff-WS maximizes the revenues from operating a large range of different backbone networks. Specifically, the obtained revenues increase as the mean nodal degree in the network gets lower.
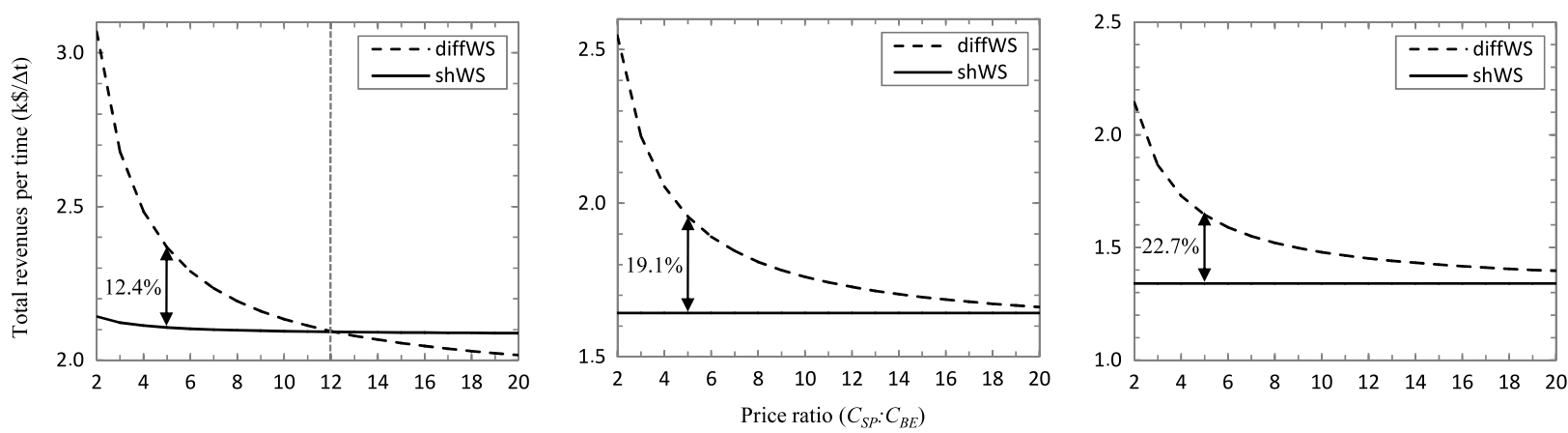

Fig. 8. Total revenues per time interval against the price ratio for the DT (left), EON (center), and NSFNET (right) base networks. The diff-WS partitioning scheme provides higher revenues than diff-WS in all networks for price ratios lower than 12:1. 


\section{ACKNOWLEDGMENTS}

The authors thank the M.Sc. student Ana Cecilia Cid for her valuable collaboration on this work. The research leading to these results has received funding from the European Community's Seventh Framework Programme FP7/2007-2013 under grant agreement no. 247674 of the STRONGEST project and from the Spanish Science Ministry through the TEC2008-02634 ENGINE project.

\section{REFERENCES}

[1] C. Ou, J. Zhang, L. H. Sahasrabuddhe, and B. Mukherjee, "New and improved approaches for shared-path protection in WDM mesh networks," J. Lightwave Technol., vol. 22, pp. 1223-1232, 2004.

[2] R. Muñoz, R. Casellas, and R. Martinez, "An experimental signalling enhancement to efficiently encompass WCC and backup sharing in GMPLS-enabled wavelength-routed networks," in Proc. IEEE ICC, 2008, pp. 5401-5406.

[3] E. Mannie and D. Papadimitriou, "Recovery (protection and restoration) terminology for generalized multi-protocol label switching (GMPLS)," IETF RFC-4427. 2006.

[4] "Terms and definitions for optical transport networks (OTN)," ITU-T Rec. G.870/Y.1352. 2010.
[5] L. Velasco, S. Spadaro, J. Comellas, and G. Junyent, "Sharedpath protection with extra-traffic in ASON/GMPLS ring networks," J. Opt. Netw., vol. 8, pp. 130-145, 2009.

[6] F. Agraz, L. Velasco, J. Perelló, M. Ruiz, S. Spadaro, G. Junyent, and J. Comellas, "Design and implementation of a GMPLS-controlled grooming-capable optical transport network," J. Opt. Commun. Netw., vol. 1, pp. A258-A269, 2009.

[7] A. Kodian and W. Grover, "Multiple-quality of protection classes including dual-failure survivable services in p-cycle networks," in Proc. IEEE BROADNETS, 2005.

[8] W. Grover and M. Clouqueur, "Span-restorable mesh networks with multiple quality of protection (QoP) service classes," Photon. Netw. Commun., vol. 9, pp. 19-34, 2005.

[9] L. Guo, J. Cao, H. Yu, and L. Li, "Path-based routing provisioning with mixed shared protection in WDM mesh networks," $J$. Lightwave Technol., vol. 24, pp. 1129-1141, 2006.

[10] M. Xia, M. Tornatore, C. Martel, and B. Mukherjee, "Servicecentric provisioning in WDM backbone networks for the future internet," J. Lightwave Technol., vol. 27, pp. 1856-1865, 2009.

[11] C. Pavan, R. Morais, J. Ferreira, and A. Pinto, "Generating realistic optical transport network topologies," J. Opt. Commun. Netw., vol. 2, pp. 80-90, 2010.

[12] CPLEX, http://www-01.ibm.com/software/integration/optimization/ cplex-optimizer/. 